\title{
Transverse Colon
}

National Cancer Institute

\section{Source}

National Cancer Institute. Transverse Colon. NCI Thesaurus. Code C12385.

The third division of the colon (large intestine). It communicates with the ascending colon in the upper right-hand quadrant of the abdomen and the descending colon in the upper left-hand quadrant. 